\title{
Corporate Governance von Finanzinstituten
}

\author{
Empirische Befunde, Theorie und Fragen in den Rechts- und \\ Wirtschaftswissenschaften
}

\author{
Klaus J. HOPT*
}

Inhaltsübersicht

ZGR 2017, 438-459

I. „Bank Governance is Special“: Zur Theorie der Corporate Governance von Finanzinstituten ..................

1. Besonderheit der Finanzinstitute gegenüber Nicht-Finanzinstituten: empirische Untersuchungen . . . . . . . . . . . . . . . . . . . . 441

2. Varianz der rechtlichen und rechtspolitischen Beiträge . . . . . . . . . . . . 444

3. Auf dem Weg zu einer Creditor Governance für Banken und Finanzinsti-

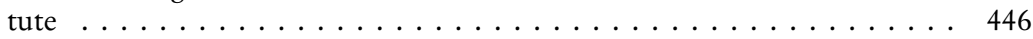

4. Folgerungen für Forschung und Reformen . . . . . . . . . . . . . . . . . . 448

II. Zur Forschungs- und Reformagenda der Corporate Governance von Finanz-

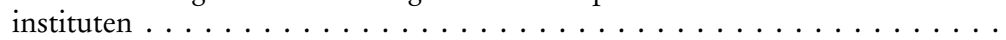

1. Corporate Governance und Transparenz . . . . . . . . . . . . . . . .

2. Zusammensetzung, Organisation und Arbeitsweise von Board bzw. Aufsichtsrat . . . . . . . . . . . . . . . . . . . . . 450

3. Organisations- und Verhaltenspflichten, Haftung . . . . . . . . . . . . . . . 453

4. Öffentlichrechtliche Finanzinstitute, insbesondere Landesbanken . . . . . . 455

5. Durchsetzung und Kontrolle durch Staat und Private . . . . . . . . . . . . 456

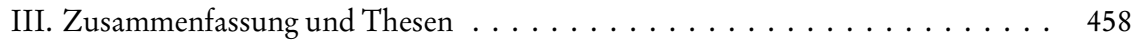

Die Corporate Governance von Finanzinstituten weist gegenüber der allgemeinen Corporate Governance ganz erbebliche Besonderheiten auf, die durch empirische Befunde belegt werden und einen umfassenden Niederschlag in der mittlerweile überbordenden, nationalen und internationalen Finanzmarktgesetzgebung, in der Aufsichtspraxis und in vielen Prinzipien und Empfehlungen gefunden haben. Dem entspricht eine eigene Theorie der Corporate Governance von Finanzinstituten, die den Interessen der Gläubiger neben oder sogar vor denen der Aktionäre Rechnung trägt. Man kann insoweit von einer Creditor (Debtholder, Depositor) Governance sprechen. Diese hat erbebliche, noch nicht ausgelotete Folgen für Forschung und eventuelle Reformen, unter anderem für den Board bzw. Aufsichtsrat, für die Organisations- und Verbaltenspflichten seiner Mitglieder, für öffentlichrechtliche Finanzinstitute und für die Durchsetzung und Kontrolle durch Staat und Private.

* Der Beitrag ist Peter Hommelhoff gewidmet, dem langjährigen Freund und Wegbegleiter in Wissenschaft und Praxis. Der Beitrag war länger ausgelegt, musste aber aus Platzgründen strikt beschränkt werden. Die ausgegliederten Teile - Corporate Governance von Finanzinstituten, Aktuelle Entwicklungen in der Praxis - werden separat veröffentlicht. 
The corporate governance of financial institutions is very special as compared to the general corporate governance of corporations. This is shown by empirical research and has led to a mass of national and financial legislation, supervisory practice, principles and recommendations. This should lead to a genuine theory of corporate governance of financial institutions that takes into account the interest of the creditors besides or even before those of the shareholders. An appropriate term for this would be creditor (debtholder, depositor) governance. This has considerable consequences for research and possible reforms that are still to be analyzed and developed. Problem areas are for example the bank board viz. the supervisory board, the duties of organization and behavior of the bank directors, the financial institutions that are owned or controlled by the state or public entities and the enforcement and control by the state and private actors.

„Effective corporate governance is critical to the proper functioning of the banking sector and the economy as a whole." So beginnen die Guidelines des Basel Committee on Banking Supervision, der weltweit tonangebenden Instanz für Bankenregulierung und Bankaufsicht, zu den Corporate Governance-Prinzipien für Banken 2015. ${ }^{1}$ Eine allgemein akzeptierte Definition von Corporate Governance gibt es zwar nicht, aber im Kern ist man sich mit der Cadbury Commission 1992 international einig, es geht um „the system by which companies are directed and controlled".2 Corporate Governance speziell von Banken und anderen Finanzinstituten ${ }^{3}$ ist im wesentlichen erst nach

1 Basel Committee on Banking Supervision, Guidelines, Corporate governance principles for banks, Bank for International Settlements (BIZ), Basel, July 2015, p. 3.

2 Zur Vergleichenden Corporate Governance, Forschung und internationale Regulierung, Hорт ZHR 175 (2011) 444 (448); englische Version IDEm, The American Journal of Comparative Law 59 (2011) 1-73. Zur Definition des Basel Committee auf der Basis der agency theory und unter Einschluss auch anderer stakeholder als der Aktionäre, Basel Committee (Fn. 1) p. 1 Glossary, unten I 3. Andere sprechen von internal governance, z.B. European Banking Authority (EBA), Consultation Paper, Draft Guidelines on internal governance, 28.10.2016. Die internal governance steht im Gegensatz zur externen Corporate Governance: disziplinierende Wirkung vor allem des Markts für Unternehmenskontrolle und anderer Märkte auf die Direktoren, näher HopT, ebenda 450. Für Banken spielt der Markt für Unternehmenskontrolle keine maßgebliche Rolle, ADams/ Mehran, Is corporate governance different for bank holding companies? Federal Reserve Bank of New York Economic Policy Review (2003) 9(1) 123 at 126; de Haan/Vlahu (Fn. 19) at 232 et seq. Zu einzelnen Fällen (ABN AMRO/Banca Antonveneta 2005, RBS/ ABN AMRO 2007, BNP/Société Générale 1999) zutreffend Tsagas, The Market For Corporate Control in the Banking Industry, in Chiu, ed., The Law on Corporate Governance in Banks, 2015, p. 285, gegen shareholder primacy und für mehr Staatskontrolle, aber ohne dass das dann drohende Protektionismusproblem gelöst wird. Zu den Control Transactions Davies/Hopt/Ringe in Kraakman et al., The Anatomy of Corporate Law, Oxford, 3 d ed. 2017, p. 205 et seq. (zit: Anatomy).

3 Der Begriff ist ökonomisch und breiter als die Definitionen in $\mathbb{S} \$ 1$ Abs. 1, 1a, 1b, III KWG und umfasst Versicherungsinstitute und andere Finanzintermediäre. Banken wird hier auch pars pro toto für Finanzinstitute gebraucht, der Schwerpunkt liegt allerdings bei Banken im engeren Sinne. 
der Finanzkrise in den Blickpunkt geraten ${ }^{4}$ und mittlerweile ein eigener internationaler, ökonomischer und juristischer Forschungsschwerpunkt. ${ }^{5} \mathrm{Ob}$ die Finanzkrise selbst eine wesentliche Ursache in Defiziten der Corporate Governance von Finanzinstituten hatte, ist sehr zweifelhaft, ${ }^{6}$ aber sie war Anlass für viele internationale Institutionen zu Empfehlungen, Aufsichtsmaßnahmen und Regulierungen von Banken und ihrer Corporate Governance, diese mittlerweile, von der Literatur noch wenig beachtet, in zweiter oder sogar dritter Generation: z.B. Basel Committee (Juli 2015), ${ }^{7}$ schweizerische FINMA (September 2016), ${ }^{8}$ EBA (Oktober 2016/Juli 2017), ${ }^{9}$ International Association of

4 Ausführlich Hopt, Journal of Corporate Law Studies 13 (2013) 219, auch in Miller, ed., Economics of Financial Law, vol. II Part III Governance, 2016, p. 573; Hорт/WoHLmannstetter, Hrsg., Handbuch Corporate Governance von Banken, 2011; Chiu 2015 (Fn. 2); Armour/Awrey/Davies/Enriques/Gordon/Mayer/Payne, Principles of Financial Regulation, Oxford 2016, ch. 17: Bank Governance; BARTH/Levine, eds., Regulation and Governance of Financial Institutions, Cheltenham 2016; Mallin, ed., Handbook on Corporate Governance of Financial Institutions, Cheltenham 2016.

5 Von 270 ökonomischen und juristischen Beiträgen der ECGI Working Paper Series des European Corporate Governance Institute (ECGI) betrafen rund die Hälfte Corporate Governance-Fragen und mehr als ein Viertel davon solche der Regulierung und Corporate Governance von Banken (im weiteren Sinn), Merkt/Klausmann, EWS 2016, 253 (254ff).

6 Hорт, Corporate Governance of Banks after the Financial Crisis, in Wymeersch/Hopt/ Ferrarini, eds., Financial Regulation and Supervision, A Post-Crisis Analysis, Oxford 2012, p. 337 at 11.16 et seq.; Berger/Imbierowicz/Rauch, The Roles of Corporate Governance in Bank Failures during the Recent Financial Crisis, Journal of Money, Credit and Banking 48 (2016) 729, bei http://ssrn.com/abstract=2021799; BINDER ZGR 2015, 667 (697) f; KотZ/Sснмidt ZBB 2016, 427 (440); Siems/Macotela (Fn. 12) 327; auch schon Cheffins, Did Corporate Governance „Fail“ during the 2008 Stock Market Meltdown? The Case of the S\&P 500 (2009) 65 Business Lawyer 1; auch Konzelmann/ Fovargue-Davies, eds., Banking Systems in Crisis: The Faces of Liberal Capitalism, Routledge 2012. Anders Mülbert/Wilhelm, CRD IV Framework for Banks' Corporate Governance, in Busch/Ferrarini, eds., European Banking Union, Oxford 2015, 155 at 6.74 et seq. Die internationalen Institutionen, die mit dieser These auch ihre eigenen Initiativen gerechtfertigt hatten, sind mittlerweile vorsichtiger geworden, z.B. EBA (Fn. 2) p. 6: „not a direct trigger for the financial crisis“. Dass Corporate GovernanceDefizite aber bei der Krise zutage getreten sind („closely associated with it“) und falsche Anreize gesetzt waren, ist unbestritten, Auflistung bei Норт, ebenda, 11.17 et seq. m.w. N. Vgl. Summers, International Financial Crises: Causes, Prevention, and Cure, American Economic Review 90(2) (2000) 1.

7 Oben Fn. 1.

8 Eidgenössische Finanzmarktaufsicht FINMA, Bern, Rundschreiben 2017/1, Corporate Governance - Banken: Corporate Governance, Risikomanagement und interne Kontrollen bei Banken, 22.9.2016.

9 Oben Fn. 2. Auch schon EBA Guidelines on the Assessment of the Suitability of Members of the Management Body and Key Function Holders of 22.9.2012 und Konsultation zu "fit and proper" durch die EBA und die European Central Bank (ECB) 2016. Dazu schon Норт (Fn. 4) at 230 et seq. 
Insurance Supervisors (November 2015), ${ }^{10}$ Financial Stability Board (April $2017)^{11}$ und, allgemein für Unternehmen, OECD (September 2015) ${ }^{12}$ und z.B. der Dutch Banking Code (Januar 2015). ${ }^{13}$

\section{I. „Bank Governance is Special“: Zur Theorie der Corporate Governance von Finanzinstituten}

\section{Besonderheit der Finanzinstitute gegenüber Nicht-Finanzinstituten: empirische Untersuchungen}

Dass Banken wesentliche Besonderheiten gegenüber Nichtbanken aufweisen, ist seit langem Allgemeingut und Grundlage für die Bankenregulierung und -aufsicht. Die Besonderheiten liegen an der gegenüber Nichtbanken sehr niedrigen Kapitalausstattung der Banken, zumal wenn sie Fristen transformieren, an der Komplexität und schwierigen Durchschaubarkeit ihres Geschäfts und ihrer Struktur, an der besonderen Vertrauensabhängigkeit und der Gefahr von Runs und an ihrer zentralen Wichtigkeit für die Wirtschaft und ihrer makroökonomischen Funktion und als Folge davon ihrer staatlichen Regulierung. ${ }^{14}$ Dies zeigt sich besonders in den immer wieder auftauchenden Bankenkrisen und den als „too big to fail, too interconnected to fail“ bezeichneten Strukturproblemen, die immer wieder staatliche Rettungen notwendig machen, wenn der bail-in nicht vorgesehen ist oder nicht funktioniert. ${ }^{15}$ Doch beschränken

10 International Association of Insurance Supervisors (IAIS), Insurance Core Principles, updated November 2015. Auch schon OECD Guidelines on Insurer Governance, 2005, revised 2011.

11 Financial Stability Board (FSB), Thematic Review on Corporate Governance, Peer Review Report, 28 April 2017, dort p. 3: Primary objective is the application „to publicly listed regulated financial institutions (e.g. banks, insurers, asset managers and financial holding companies)“.

12 G20/OECD Principles of Corporate Governance, OECD Report to G20 Finance Ministers and Central Bank Governors, Paris, September 2015. Dazu Siems/AlvarezMacotela, The G20/OECD Principles of Corporate Governance 2015: A Critical Assessment of their Operation and Impact, Journal of Business Law 2017, 310.

13 Dutch Banking Code, $2 \mathrm{~d}$ version, in effect on January 1, 2015.

14 Kotz ZVglRWiss 113 (2014) 470 (471ff); KотZ/Sснмidt ZBB 2016, 427 ff; Kоккinis, A Primer on Corporate Governance in Banks and Financial Institutions: Are Banks Special? in Chiu (Fn. 2) p. 1 (9 et seq.).

15 Dazu außer Wymeersch et al. (Fn. 6) Ferran/Moloney/Hill/Coffee, eds., The Regulatory Aftermath of the Global Financial Crisis, Cambridge 2012, und darin speziell zu den USA Coffee p. 301 et seq; Faia/Hackethal/Haliassos/Langenbucher, eds., Financial Regulation, A Transatlantic Perspective, Cambridge 2015; Hui Huang/SchoenMAKER, eds., Institutional Structure of Financial Regulation, Theories and international experiences, London 2015. Allgemeiner WitTMAn, ed., The Economics of Governance, 2017. 
sich diese Besonderheiten der Banken nicht auf die systemisch relevanten Banken (systemically important banks, SIFIs), sondern gelten allgemeiner, auch wenn sie natürlich für die SIFIs besonders folgenträchtig und sichtbar sind. ${ }^{16}$

Dass die Besonderheit der Banken auch eine besondere Corporate Governance verlangt, ist demgegenüber erstaunlicherweise erst spät zur Kenntnis genommen worden. ${ }^{17}$ Das hat mehrere Ursachen. Die empirische, meist US-amerikanische Literatur war, auch soweit der Fokus ausnahmsweise auf Banken abzielte, meist auf die Prinzipal-Agenten-Theorie und den Konflikt zwischen Direktoren und Aktionären ausgerichtet, wie das der US-amerikanischen Aktionärsstruktur (Streubesitz) entsprach. ${ }^{18}$ Untersuchungsgegenstand waren dann schon aus Gründen der Verfügbarkeit von Daten in aller Regel börsennotierte Gesellschaften. Erst in jüngerer Zeit mehren sich empirische Untersuchungen speziell zur Corporate Governance von Banken, die die Besonderheit auch ihrer Corporate Governance belegen. Hier können nur einige wichtige Befunde erwähnt werden. ${ }^{19}$

16 Statt aller Admati/Hellwig, The Bankers' New Clothes, What's Wrong with Banking and What to Do about It, Princeton 2013.

17 Mit am frühesten Corrigan, Are Banks Special? (1982) Annual Review (The Federal Reserve Bank of Minneapolis); Fama, What's Different about Banks? (1985) 15(1) Journal of Monetary Economics 29; auch die special issue: Corporate Governance: What Do We Know, and What is Different about Banks?, (2003) 9 Economic Policy Review (The Federal Reserve Bank of New York, FRBNY) 1.

18 KотZ/Sснмidt ZBB 2016, 427 (430 et seq.), die demgegenüber die Theorie der Nexuse unvollständiger Verträge und die der varieties of capitalism betonen, letzteres im Anschluss an Hall/Soskice, eds., Varieties of Capitalism: The Institutional Foundations of Comparative Advantage, Oxford 2001.

19 Näher schon Hopt (2013, Fn. 4) at 239-244; Hagendorff, Corporate Governance in Banking, in Berger/Molyneux/Wilson, eds., The Oxford Handbook of Banking, $2 \mathrm{~d}$ ed. 2015, p. 139; umfassender Bericht über die empirische Literatur zuletzt bei DE HAAN/ Vlahu, Corporate Governance of Banks: A Survey, Journal of Economic Surveys 30 (2016) No 2, p. 228-277; kurz auch FERrarini, Understanding the Role of Corporate Governance in Financial Institutions: A Research Agenda, ECGI Law Working Paper No 347/2017, March 2017, p. 7 et seq. Neuerdings z.B. Hamdani, Bank Directors: Duties Towards the Public? und Tröger, Managers' Duties Towards Shareholders and Debtholders, Society of European Contract Law (SECOLA), 16-17.6.2017 Bocconi University Milan. Zu den Beiträgen in ECGI Merkt/Klausmann (Fn. 5). Einschränkend jedoch van der Elst, Corporate Governance and Banks: How justified is the match? February 2015, bei http://ssrn.com/abstract=2567072, der aber am Ende p. 32 dann doch zugibt, dass bank governance speziell ist. Die allgemeinen Feststellung durch Untersuchungskommissionen - in UK etwa zur Royal Bank of Scotland und zur Halifax Bank of Scotland, Walker-ArnotT, Corporate Governance and banks: The role and composition of the board, in Chiu (Fn. 2), p. 42 at p. 55 et seq. -, dass die bank boards versagt haben, sind richtig, aber besagt für die These hier für sich noch nichts. 
Fahlenbrach und Stulz ${ }^{20}$ berichten, dass Banken-CEO, bei denen die Anreize stärker auf die Interessen der Aktionäre ausgerichtet waren, schlechtere Ergebnisse erzielten. Ähnliche Ergebnisse fanden Beltratti und Stulz ${ }^{21} \mathrm{zu}$ den Boards von Banken. Banken mit Boards, die aktionärsfreundlicher agierten, hatten signifikant schlechtere Ergebnisse. Nach anderen Studien hatten die Zusammensetzung und Charakteristika der Boards von Banken wesentliche Auswirkungen ${ }^{22}$ und Banken gingen mehr und höhere Risiken ein, wenn die Aktionäre stärker im Board vertreten sind. ${ }^{23}$ Offenbar haben die Bank-Boards sich mehr an die Präferenzen der Aktionäre gehalten, ${ }^{24}$ die, wenn hinreichend diversifiziert, risikofreudiger sind als etwa die Gläubiger. ${ }^{25}$ Beltratti und Stulz bezweifeln deshalb die These, dass eine schlechte Corporate Governance der Banken eine wesentliche Ursache für die Finanzkrise war. ${ }^{26}$ Banken mit unabhängigen Boards sind schlechter gefahren. ${ }^{27}$ Banken, die von Aktionären kontrolliert wurden, hatten vor der Krise höhere Gewinne im Vergleich zu Banken, die vom Management kontrolliert waren. ${ }^{28}$ Unternehmen, bei denen institutionellen Anleger Aktien hielten, fuhren entsprechend schlechter. ${ }^{29}$ Allgemeiner zeigte sich, dass die Aktionärsstruktur der Bank einen starken Ein-

20 Fahlenbrach/Stulz, Bank CEO Incentives and the Credit Crisis, (2011) 99 Journal of Financial Economics 11.

21 Beltratti/Stulz, The Credit Crisis Around the Globe: Why Did Some Bank Perform Better? (2012) 105 Journal of Financial Economcis 1; auch Ferreira/Kershaw/KirchMAier/Schuster, Shareholder Empowerment and Bank Bailouts, November 2012, bei ht tp://ssrn.com/abstract=2170392 m.w.N. Insoweit zustimmend auch vAN DER ELST (Fn. 19), aber unter Hinweis, dass die Zusammensetzung von boards nicht nur zwischen Banken und anderen Unternehmen, sondern auch nach Ländern unterschiedlich ist.

22 Spong/Sullivan, Bank Ownership and Risk Taking: Improving Corporate Governance in Banking after the Crisis, September 2010, bei http://ssrn.com/abstract=1900609.

23 Pathan, Strong Boards, CEO Power and Bank Risk-taking, (2009) 33(7) Journal of Banking and Finance 1340.

24 Vgl. Agarwal/Erel/Stulz/Williamson, Differences in Governance Practices between US and Foreign Firms: Measurement, Causes, and Consequences, (2009) 22/8 Review of Financial Studies 3131.

25 Laeven/Levine, Bank Governance, Regulation, and Risk Taking, (2009) 93 Journal of Financial Economics 259; Ferreira/Kershaw/Kirchmaier/Schuster (Fn. 21).

$26 \mathrm{Zu}$ dieser umstrittenen Frage schon oben bei Fn. 6.

27 Erkens/Hung/Matos, Corporate Governance in the 2007-2008 Financial Crisis: Evidence from Financial Institutions Worldwide, Journal of Corporate Finance 38 (2012) 389; Pathan (Fn. 23). Vgl. Ferreira/Kirchmaier/Metgzer, Boards of Banks, September 2012, bei http://ssrn.com/abstract=1620551. Weitere Nachweise zu neutralen, negativen und ausnahmsweise positiven Relationen in empirischen Studien bei de HaAN (Fn. 19) at $251-254$.

28 Gropp/KöHler, Bank Owners or Bank Managers: Who is Keen on Risk? Evidence from the Financial Crisis, February 2010, bei http://ssrn.com/abstract=1555663.

29 Erkens/Hung/Matos (Fn. 27). 
fluss auf ihre Insolvenz hatte, besonders wenn rangniedrigeres Management wesentlich beteiligt war. ${ }^{30}$

Diese und weitere empirische Untersuchungen legen nahe, dass manche herkömmliche, auch empirisch begründete Annahmen zu guter Corporate Governance bei Unternehmen auf die Corporate Governance von Banken nicht ohne weiteres übertragen werden können, ja hier sogar Gegenteiliges gelten kann. Das ist etwa der Fall für die Unabhängigkeit von Direktoren, die allerdings nach neuesten Studien auch sonst auch negative Auswirkungen haben kann, ${ }^{31}$ während Sachkunde und Erfahrung demgegenüber mehr zählen, dies jedenfalls wenn klare Interessenkonflikte vermieden werden. ${ }^{32}$ Allgemeiner ist aber aus verschiedenen Gründen Augenmaß bei Schlüssen aus den empirischen Befunden angezeigt. Häufig stehen sich differenzierte Ergebnisse gegenüber, was an unterschiedlichen Zeithorizonten der Untersuchungen, einer nicht hinreichenden Berücksichtigung von Interdependenzen bestimmter Faktoren und vor allem an länder- und pfadabhängigen Unterschieden auf Grund von rechtlichen Regelungen und kulturellen Gegebenheiten liegen kann. ${ }^{33}$

\section{Varianz der rechtlichen und rechtspolitischen Beiträge}

Die rechtlichen und rechtspolitischen Beiträge zur Corporate Governance von Banken sind sehr unterschiedlicher Art und können hier nicht näher behandelt werden..$^{34}$ Ein erheblicher Teil befasst sich mit den europarechtlichen Vorgaben der Capital Requirements Directive (CRD IV) und von Solvency II und ihren

30 Berger/Imbierowicz/Rauch (Fn. 6). Zu den verschiedenen empirischen Studien mit teilweise unterschiedlichen Ergebnissen zu concentrated shareholdership, shareholdership of CEO and directors und government ownership de HAan/VlaHU (Fn. 19) at 256 et seq.; auch Hagendorff (Fn. 19) p. 151 et seq.; auch Mamatzakis/Bermpei, The Effect of Corporate Governance on the Performance of US Investment Banks, Financial Market Institutions \& Instruments (New York University) 24 (2015) 191. Anders vaN DER Elst (Fn. 19).

31 Cavaco/Crifo/Rebérioux/Roudant, Independent director: Less informed but better selected than affiliated board members? Journal of Corporate Finance 43 (2017) 106.

32 Unten II 2.

33 Das räumen auch De HaAn/Vlahu (Fn. 19) at 266 ein. Auf die Besonderheiten des deutschen Drei-Säulen-Bankensystems weisen KотZ/SснміDт ZBB 2016, 427 (428ff) hin, dies auch nach dem Abbau der „Deutschland AG“ und des herkömlichen rheinischen Kapitalismus, ebenda 434, $437 \mathrm{ff}$. Zum Letzteren Ringe, Changing Law and Ownership Patterns in Germany: Corporate Governance and the Erosion of Deutschland AG, The American Journal of Comparative Law 63 (2015) 493.

34 Zur Literatur bis 2013 Норт (Fn. 4) at p. 220 n. 3. Zur internationalen, insbesondere ökonomischen Literatur Merkt/Klausmann (Fn. 5), dort auch Aufzählung und zum Stand der deutschen Corporate Governance-Diskussion S. 270, dort Fn. 211 zur Corporate Goverance von Banken. 
Umsetzungen vor allem zu den Organen Aufsichtsrat/Vorstand/Geschäftsleiter in $\$ 25 \mathrm{~d}$ KWG und $\$ 24$ VAG, teils rein de lege lata Europaea et Germanica, ${ }^{35}$ teils eher funktional-rechtspolitisch. ${ }^{36}$ Interessant ist dabei das Zusammenspiel von Aktien-, Bankaufsichts- und Versicherungsaufsichtsrecht bei der Corporate Governance. ${ }^{37}$ Andere bemerken zu Recht, dass sich allmählich ein eigenes Bankgesellschafts ${ }^{38}$ bzw. Bankaktienrecht ${ }^{39}$ herausbildet, und fragen sich, welche Auswirkungen die Europäische Bankenunion auf die Governance von Kreditinstituten haben $\operatorname{wird}^{40}$. Die gegenseitigen Einflüsse können weiterführen, Brüche sind nicht ausgeschlossen, lassen sich aber in der Regel überwinden. Die Parallele zur Diskussion um ein Börsengesellschaftsrecht, das auf dem Erfurter Juristentag 2008 kontrovers diskutiert wurde ${ }^{41}$ und in anderen Rechtsordnungen wie der Schweiz und Österreich bereitwilliger akzeptiert wird, liegt auf der Hand. Weiterungen ergeben sich für ein eigenes Recht der Finanzintermediäre und ihre Corporate Governance. ${ }^{42}$ In der internationalen Literatur findet sich teils extreme Skepsis auf der Basis grundsätzlich anderer Vorstellungen zu Regulierung, ${ }^{43}$ teils wird die zu geringe Berück-

35 Z.B. Boos/Fischer/Schulte-Mattler, KWG CRR-VO, Bd. 1, 5. Aufl. 2016, \25d KWG; Reischauer/Kleinhans, KWG, LBl, \25 d KWG; Velte/Buchiolz ZBB 2013, 400 (CRD IV); Kumpan/Pauschinger EuZW 2017, 327 (331, kurz zur Reform von CRR/CRD IV); Brandi NZG 2012, 1321 (KWG); Leuschner/Wolfgarten WPg 2015, 375 (KWG); Schneider/Schneider NZG 2016, 41 (KWG); Bürkle WM 2012, 878 (Solvency II); Dreher/Ballmaier ZGR 2014, 753 (Solvency II); Hersch VersR 2016, 145 (\$24 VAG); Krimphoven VersR 2017, 326 (VAG, BaFin-Rundschreiben MaGo); Bronnert-Härle, Aufsichtsratsbeschlüsse als neue Akteure der internen Corporate Governance von Banken, 2016 (mit Auswirkungen des $\$ 25 \mathrm{~d}$ KWG).

36 Mülbert/Wilhelm (Fn. 6, CRD IV); Mülbert/Wilhelm ZHR 178 (2014) 502 (CRD IV, Risikomanagement, Compliance); Mülbert ZVglRWiss 113 (2014) 520 (Corporate Governance, CRD IV).

37 Leyens/SCHMidt AG 2013, 533.

38 LANGENBUCHER ZHR 176 (2012) 652.

39 Zetzsche/Noncк, Bankaktienrecht und Aktienbankrecht, in Grundmann ua, Festschrift für Hopt, 2010, Bd. 2, S. 2283.

40 Binder, The Banking Union and the Governance of Credit Institutions: A Legal Perspective, Eur.Bus.Org. Law Rev. (2015) 16:467, in particular general prudential requirements and remuneration, at 479 et seq.

41 BAYER, Empfehlen sich besondere Regeln für börsennotierte und für geschlossene Gesellschaften? Gutachten E zum 67. Deutschen Juristentag Erfurt 2008; Richter ZHR 172 (2008) 419.

42 Dazu Leyens, Informationsintermediäre des Kapitalmarkts, Private Marktzugangskontrolle durch Abschlussprüfung, Bonitätsrating und Finanzanalyse, Hamburger Habilitationsschrift 2015, Tübingen (im Druck).

43 Z.B. Romano, For Diversity in the International Regulation of Financial Institutions: Critiquing and Recalibrating the Basel Architecture, 31 Yale Journal of Regulation 1 (2014); Enriques/Zetzsche, Quack Corporate Governance, Round III? Bank Board Regulation Under the New European Capital Requirement Directive, ECGI Law 
sichtigung des two-tier Systems bemängelt. ${ }^{44}$ Hinzu kommen vielfältige Einzelvorschläge etwa zu strengerer Haftung der Direktoren. ${ }^{45}$ Auffällig ist die Diskrepanz zwischen immer detaillierteren, mehr oder weniger verbindlichen Vorgaben durch Gesetzgeber, Aufsichtsbehörden und internationale Gremien $^{46}$ einerseits und die eine Überregulierung beklagende Praxis. Mit letzterer stimmen weite Teile der Literatur mit Recht überein. ${ }^{47}$

\section{Auf dem Weg zu einer Creditor Governance für Banken und Finanzinstitute}

Für die Theorie der Corporate Governance von Banken und Finanzinstituten ergibt sich aus den empirischen Befunden, den Erfahrungen aus der Finanzkrise und den wirtschafts- und rechtswissenschaftlichen Stellungnahmen ein Perspektivenwandel von einer ausschließlichen (USA) oder jedenfalls im Hinblick auf den Unternehmensbestand und eine dauerhaftige Rentabilität vorrangigen (Europa) Shareholder-Orientierung ${ }^{48}$ hin zu einer Theorie der Creditor (Debtholder, Depositor) Governance. ${ }^{49}$ Für die Sanierung und Insolvenz nicht nur

Working Paper No 249/2014, March 2014, und in Theoretical Inquiries in Law 16(1) 211, im Titel und in der Grundhaltung Annäherungen an Romano, The Sarbanes-Oxley Act and the Making of Quack Corporate Governance, Yale Law Journal 114 (2005) 1521. $\mathrm{Zu}$ dieser zutreffend, aber zu hart die Kritik von Coffee (Fn. 15) at 306 and passim. Vgl. auch Pargendler, The Corporate Governance Obsession, 42 Journal of Corporation Law 101 (2016).

44 Unter vielen Mülbert ZVglRWiss 113 (2014) 520 (526ff).

45 Armour/Gordon, Systemic Harms and Shareholder Value, ECGI Law Working Paper No 222/2013, August 2013.

46 Oben Fn. 7-13.

47 Statt vieler FERRARini (Fn. 19) p. 19, 24: für einen supervisory approach statt eines regulatory approach, das entspricht dem Unterschied zwischen principle-based und rule-based regulation. Vgl. auch zu rules and standards Anatomy (Fn. 2), p. 32 et seq. m.w.N. Auch Mülbert/Wilhelm (Fn. 6) at 6.83 sprechen von 70 delegated and implementing acts zu CRD IV.

48 Vgl. dazu die Modifikationen in Anatomy 3d ed. 2017 (Fn. 2) 1.5 at 22-24 gegenüber Anatomy $2 \mathrm{~d}$ ed. 2009, 1.5 at 28-29, die nach eingehenden Diskussionen konsentiert wurden: „(S)hareholder value is the proper object of corporate law“, weil die Aktionäre die „residual claimants“ sind und das ein klarerer Yardstick für die Beurteilung der Leistung der Direktoren ist, was selbstverständlich nicht Profitmaximierung um jeden Preis bedeutet. Für ein sozial orientiertes Gesellschaftsrecht aber z.B. PARgENDLER (Fn. 43). Zur deutschen Diskussion zusammenfassend HüFfER/KoCH, Aktiengesetz, 12. Aufl. 2016, $\$ 76$ Rn $28 \mathrm{ff}$; der Vorstand hat danach die divergierenden Interessen nach dem Gebot der praktischen Konkordanz abzuwägen, Hopt ZGR 1993, 534 (536 f), ZGR 2002, 333 (360), HÜFFER/KOCH $\$ 76$ Rn 33.

49 Näher dazu Норт (Fn. 4) at 243 et seq.; Koккinis (Fn. 14) p. 35 et seq. Aus wirtschaftswissenschaftlicher Sicht schon MaceY/O’HaRA, The Corporate Governance of Banks, 
von Banken ist schon länger anerkannt, dass dabei Herrschaft und Risiko von den Eigentümern zu den Gläubigern übergehen. ${ }^{50}$ Das Basel Committee on Banking Supervision formuliert in seinen maßstabssetzenden Corporate governance principles for banks vom Juli 2015 gleich zu Beginn: „The primary objective of corporate governance should be safeguarding stakeholders' interest in conformity with public interest on a sustainable basis. Among stakeholders, particularly with respect to retail banks, shareholders' interest would be secondary to depositors' interest. " ${ }^{51}$ Das ist eine klare Absage, die von nationalen und internationalen Bankaufsichtsbehörden geteilt wird, an die in der allgemeinen Corporate Governance-Literatur vorherrschende Auffassung von einem, wenngleich in Europa Stakeholderinteressen ebenfalls berücksichtigenden Primat der Aktionärsinteressen..$^{52}$ Das führt zugleich zu einer Relativierung der Bedeutung von controlling shareholders, institutional investors und allgemeiner shareholder control, wie sie heute für die Corporate Governance von Unternehmen im Zentrum der Aufmerksamkeit stehen. Allerdings ist damit nicht gesagt, dass diese spezielle Stakeholder Governance über den Sektor der Banken und Finanzinstitute hinaus in die allgemeine Theorie der Corporate Governance von Aktiengesellschaften übernommen werden müsste. ${ }^{53}$

Damit stellt sich aber zugleich die Frage nach dem Einfluss des Staates auf die Corporate Governance von Banken, die zumal was mögliche politische Einflüsse bei öffentlichrechtlichen Finanzinstituten angeht, durchaus problematisch ist. ${ }^{54}$ Für die Corporate Governance von Banken ist vorgeschlagen wor-

(2003) 9 Economic Policy Review (FRBNY) 91; Kотz/Schmidt ZBB 2016, 427 (440): „(S)hareholder governance needs to be complemented by ... depositor or creditor governance." Debt governance allein reicht allerdings nicht aus, sondern staatliche Regulierung, insbesondere durch Anforderungen an Kapital (vgl Admati/Hellwig, Fn. 16) und Durchsetzung, sind notwendig, unten II 5 und schon Dewatripont/Tirole, The prudential regulation of banks, MIT1994. Insoweit auch VAN DER ELST (Fn. 19) at p. 32, aber zuvor mit dem Hinweis, debtholders hätten keine Einfluss. Der Begriff ist hier aber breiter für Gläubiger verwandt, also einschließlich der Schuldverschreibungsgläubiger (bondholders), im übrigen plädiert van der Elst aber selbst für mehr Einfluss von debtholders (depositors and bondholders), at p. 32.

50 Anatomy (Fn. 2) at 127 et seq.: Distressed firms, und schon 114: The vicinity of insolvency. Ferner z.B. Schenker, Corporate Governance in Sanierungsfällen - Der Einfluss der Gläubiger: Chancen und Risiken, Festschrift von der Crone, Zürich 2017, S. 65. Dabei spielt das Risiko der Banken, bei Eingreifen als faktisches Organ mit entsprechenden Pflichten- und Haftungsfolgen behandelt zu werden, eine abschreckende Rolle.

51 Basel Committee (Fn. 1) p. 3, Introduction No. 2.

52 Dazu näher Норт ZHR 175 (2011) 444 (449, $494 \mathrm{ff})$.

53 Ferrarini (Fn. 19) p. 19 et seq.: justified only from a regulatory viewpoint and not to be accepted in corporate law. Auch Anatomy 3 d ed. 2017 (Fn. 2).

54 Dazu unten II 4. Zu dem anderen Verständnis von Banken als service public in Frankreich und den Rechtsfolgen kritisch schon Hopt ZHR 143 (1979) 139. 
den, diese Verschiebung hin zu einer Creditor Governance theoretisch mit einer Erweiterung des Prinzipal-Agenten-Konflikt zu einem dreiseitigen unter Einschluss des Staates zu erfassen. ${ }^{55}$ Das trägt zwar dem Einfluss der Staatsaufsicht Rechnung, weicht aber von der inzwischen auch international anerkannten Prinzipal-Agenten-Theorie mit drei Agenturproblemen (shareholder/manager, controlling shareholder/minority shareholders, shareholders/creditors) ${ }^{56}$ wesentlich ab. Vor allem aber wird die Behandlung von Gesetzgeber und Aufsichtsbehörden als bloßem weiteren Prinzipal der Regelungs- und Aufsichtsaufgabe des Staates nicht gerecht, denn dieser muss die drei Agenturprobleme letztlich mit seiner Autorität (nach deutschen öffentlichrechtlichen Vorstellungen in einem Über- und Unterordnungsverhältnis) verbindlich lösen. ${ }^{57}$

\section{Folgerungen für Forschung und Reformen}

Mit der theoretischen Erfassung der Corporate Governance von Banken und Finanzinstituten als Creditor Governance stellt sich die Aufgabe, die verschiedenen Corporate Governance-Probleme, die in der internationalen Praxis und Literatur für Unternehmen umfassend diskutiert werden, ${ }^{58}$ darauf hin durchzumustern, was daraus für die Corporate Governance von Banken und Finanzinstituten konkret folgt bzw. wo Fragen und Lösungsvorschläge gleich bleiben oder sich verschieben. Das ist eine umfassende Aufgabe, für die hier nur einige Wegmarken und unter II. einige Beispiele angegeben werden können. Zunächst geht es dabei sicher um die Aufarbeitung der gesetzlichen Vorgaben, in der Europäischen Union durch CRD IV und Solvency II und ihre nationale Umsetzungen, in Deutschland im KWG und VAG. ${ }^{59} \mathrm{Zu}$ beachten ist dabei jedoch auch, was sich aus der Fortentwicklung, wenngleich zunächst überwiegend nur empfehlender Art, durch die oben genannten Institutionen $^{60}$ ergibt. Das ist bisher zwar in der Praxis, aber soweit ersichtlich noch nicht allgemeiner in der Literatur geleistet worden. Der Fokus darf sich

55 Langenbucher, ZHR 176 (2012) 652 (663ff).

56 Anatomy (Fn. 2) 2.1 Three Agency Problems, p. 29 et seq.

57 Das schließt nicht aus, die externe Aufsicht und die Gerichte als Akteure in der Corporate Governance neben Verwaltung, Anteilseignern, Arbeitnehmern und Informationsintermediären zu betrachten, vgl. Норт ZHR 175 (2011) 444 (514ff, $461 \mathrm{ff}$ ).

58 Dazu unter vielen Beвснuк/Weisbach, The State of Corporate Governance Research, Review of Financial Studies 23(3) 2010, 939; Fleckner/Hopt, eds., Comparative Corporate Governance, A Functional and International Analysis, Cambridge 2013 und darin der General Report von Норт, Comparative corporate governance: the state of the art and international regulation, p. 3-101. Aus Deutschland Hommelhoff/Hopt/von WerDER, Hrsg., Handbuch Corporate Governance, 2. Aufl. 2009.

59 Dazu die Literatur oben unter I 2.

60 Oben vor I, Fn. 7-13. 
allerdings nicht auf Aktien- und Bankrecht beschränken, Corporate bzw. Creditor Governance ist mehr. ${ }^{61}$ Es fragt sich also, wo und auf welche Weise den Gläubigern mehr Einfluss oder auch nur den Gläubigerinteressen mehr Beachtung eingeräumt werden kann. Das sollte in einer Forschungsagenda ${ }^{62}$ Niederschlag finden, die von Ökonomen und Juristen und möglicherweise Vertretern anderer Disziplinen gemeinsam zu entwerfen und abzuarbeiten wäre. ${ }^{63}$ Parallel dazu und hoffentlich darauf zurückgreifend mag eine Reformagenda entstehen, vorsichtig Schritt für Schritt, unter Vermeidung zu vieler zwingender und kleinteiliger Vorgaben und eingedenk der enormen Probleme einer präzisen und quantifizierbaren Kosten-Nutzen-Analyse ${ }^{64}$.

\section{Zur Forschungs- und Reformagenda der Corporate Governance von Finanzinstituten}

\section{Corporate Governance und Transparenz}

Transparenz und Offenlegung sind herkömmliche Instrumente für Aktionärsund Gläubigerschutz. Beides geht meist zusammen, zumal Anleger nicht nur Aktionäre, sondern auch Gläubiger sein können und ob das eine oder das andere der Fall ist, für Unternehmen und Anleger von den verschiedensten Faktoren abhängt. Auch speziell für Banken und Finanzinstitute spielt Transparenz eine maßgebliche Rolle, hier - anders als für Unternehmen sonst - auch zur Information der Aufsicht, die dann weniger eingreifen muss oder gezielter eingreifen kann. ${ }^{65}$ Komplexe und undurchsichtige Strukturen zumal bei internationalen Bankkonzernen sind risikofördernd und im Gefolge von Bankenkrisen immer wieder Anlass zu mehr Transparenzanforderungen geworden. ${ }^{66}$

61 Dazu Gilson, From Corporate Law to Corporate Governance, ECGI Law Working Paper No 324/2016, September 2016, bei http://ssrn.com/abstract_id=2819128.

62 Aus ökonomischer Sicht z.B. HagendorfF (Fn. 19) p. 155 am Ende: „(T)hese unique features of banks call for a more profound rethink of the corporate governance of banks, one that centers around debtholders rather than equity holders".

63 Dem wird sich ein multidisziplinäres Projekt widmen: Busch/Ferrarini/van Solinge, eds., Corporate Governance of Financial Institutions: Law, Conduct and Culture, mit einer Eröffnungskonferenz in Amsterdam im Januar 2018 und einer Veröffentlichung bei Oxford University Press.

64 Letztere hält für unmöglich CoATEs, Cost-Benefit Analysis of Financial Regulation: Case Studies and Implications, ECGI Law Working Paper No. 234/2014, January 2014, p. 2 et seq., 31 et seq.

65 Z.B. Basel Committee (Fn. 1) Principle 12 Disclosure and transparency, p. 36 et seq.; EBA (Fn. 9) Title V Transparency, p. 51 et seq.

66 Auch Basel Committee (Fn. 1) p. 23 et seq.: complex or opaque structures; Hopt (Fn. 6) 11.19 p. 345 et seq., zusammen mit anderen Schwachstellen, die im Gefolge der Finanzkrise offenkundig geworden sind. 
Offenlegungswünsche und Geheimhaltungsbedürfnisse kollidieren auch hier, und Transparenz gegenüber der Öffentlichkeit hat nicht nur positive Folgen. ${ }^{67}$ Transparenz und Offenlegung sind, wenn sie den Unterschieden der Adressanten (Größe, Komplexität, Struktur, ökonomische Bedeutung, Risikostruktur u.a.) Rechnung tragen und nicht überzogen sind, akteurs- und marktfreundlichere Instrumente als eigentliche Regulierung. ${ }^{68}$ Auch wenn die Inhalte der Offenlegung je nach besonderen Informationsinteressen der Gläubiger differieren können, ergeben sich doch im Großen und Ganzen aus der Verschiebung hin zu einer Creditor Governance keine grundsätzlichen Unterschiede. ${ }^{69}$

\section{Zusammensetzung, Organisation und Arbeitsweise von Board bzw. Aufsichtsrat}

Hierzu ergeben sich die meisten Änderungen, die aber weitgehend schon vom Gesetzgeber vorgegeben sind und hier nicht näher behandelt werden können. ${ }^{70}$ Zur Zusammensetzung des Board bzw. Aufsichtsrats von Banken und Finanzinstituten $^{71}$ ist überlegt worden, ob die Creditor Governance zur Repräsentation der Gläubiger oder jedenfalls, deren Interessen wahrnehmend, der Bankaufsicht führen müsste. ${ }^{72}$ Das erinnert an frühere Überlegungen zur Aufnahme

67 Umfassend Leuz/Wysocki, The Economics of Disclosure and Financial Reporting Regulation: Evidence and Suggestions for Future Research, Journal of Accounting Research 2016, 54(2), 525.

68 Zur Transparenz als primärem Regulierungsinstrument der EU-Kommission in jüngerer Zeit Hopt, Corporate Governance in Europe: A Critical Review of the European Commission's Initiatives on Corporate Law and Corporate Governance, NYU Journal of Law \& Business 12 (2015) 139 (202 et seq.).

69 So lautet z.B. Principle 12: Disclosure and transparency: „The governance of the bank should be adequately transparent to its shareholders, depositors, other relevant stakeholders and market participants." Basel Committee (Fn. 1) p, 36 et seq.

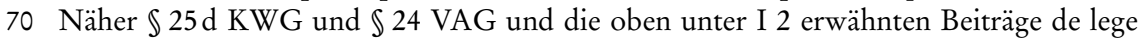
lata.

71 Empirische Untersuchungen sind meist angelsächsisch und zum one-tier board. Für Deutschland aber z.B. Johansen/Laser/Neuberger/Andreani, Insider or outside control of banks? Evidence from the composition of supervisory boards, European Journal of Law and Economics 43 (2017) 31. Empirische Befunden zur Relevanz der Größe von Boards bei de HaAn/Vlahu (Fn. 19) at 234 et seq. (eher negativ) und zur Diversität bei HagendorfF (Fn. 19) at 149 et seq., aber unklar, ob insoweit für Banken Besonderes anzunehmen ist. Interessant, aber international soweit ersichtlich singulär ist Israel, wo bei Banken die Aktionäre nur einen Direktor ernennen, während die Direktoren sonst von einem Regierungskomitee berufen werden.

72 Z.B. HagendorfF (Fn. 19) p. 155 zur Gläubigervertretung, eventuell entsprechend dem jeweiligen level of leverage; BЕснт, The Governance of Financial Institutions in Crisis, in Grundmann ua, Festschrift für Hopt, 2010, Bd. 2, S. 1615 (1625f) mit Überlegungen 
eines Vertreters des öffentlichen Interesses in den Board bzw. Aufsichtsrat, ${ }^{73}$ die $\mathrm{zu}$ Recht nicht umgesetzt worden sind. Dabei wird aber zu Recht auf die Unvereinbarkeit mit der Mitbestimmung (nur) durch die Arbeitnehmerseite in Deutschland und anderen Ländern hingewiesen, wobei allerdings zu berücksichtigen ist, dass jedenfalls die paritätische Mitbestimmung deutscher Provenienz international nicht anschlussfähig, wie schon die mangelnde Resonanz auf viel vorsichtigere Anläufe zuletzt im Vereinigten Königreich bestätigt. Die Erfahrungen mit der unternehmerischen Mitbestimmung gehen dahin, dass die Arbeitnehmer sich nicht als Interessenvertreter der Gläubiger insgesamt verstehen und gerieren, weder intern im Unternehmen noch extern als Gewerkschaften, sondern ihre eigenen, ganz spezifischen Interessen wahrnehmen. Bankenvertreter aus dem eigenen Haus und von anderen Banken sind im Aufsichtsrat von Banken gut vertreten. ${ }^{74}$ Aber auch hier ist die Erfahrung so, das die Vorstellung, die Banken als Unternehmensgläubiger würden die Interessen aller Stakeholder wahrnehmen, praxisfern ist, auch wenn das speziell im Vorfeld der Insolvenz funktional durchaus so sein kann.

Interessant ist, dass nach den empirischen Befunden ${ }^{75}$ die Unabhängigkeit von Bank-Board- bzw. -Aufsichtsratsmitgliedern gegenüber Sachkunde und Erfahrung deutlich weniger bedeutsam ist, von Interessenkonflikten abgesehen. Das läuft der herkömmlichen Meinung zur Corporate Governance von Unternehmen zuwider und ist angesichts der Besonderheiten der Transparenz und anderen Probleme bei Banken und Finanzinstituten auch einleuchtend. ${ }^{76}$ Eine Ausnahme davon scheint für Prüfungs- und Risikoausschüsse zu gelten, für die auch Unabhängigkeit eine größere Rolle spielt. ${ }^{77}$ Allerdings ist auch für Unternehmen allgemein zu beobachten, dass der aus den USA und Großbritannien, also aus Ländern mit Aktionärsstreubesitz kommende Glaube in die Unabhängigkeit von Direktoren als Allheilmittel inzwischen deutlich abgenommen hat. ${ }^{78}$ Die einschlägigen Empfehlungen und die Aufsicht tragen

zur Vertretung von Gläubigern bzw. Gläubigerinteressen durch Einlagesicherungsinstitutionen wie in den USA der Federal Deposit Insurance Corporation (FDIC).

73 Z.B. Bericht der Sachverständigenkommission zur Auswertung der bisherigen Erfahrungen bei der Mitbestimmung (Mitbestimmungskommission), BT-Drucks. VI/334, 1970, S. $107 f$.

74 Johansen et al. (Fn. 71).

75 Erkens/Hung/Matos (Fn. 27) und weitere Untersuchungen bei De Haan/Vlahu (Fn. 19) at 251 et seq. Auch Nguyen/Hagendorff/Eshraghi, Can Bank Boards Prevent Misconduct? Review of Finance 2016, 1 (31).

$76 \mathrm{Zu}$ den deutschen Landesbanken erst unten Hau/Thum (Fn. 103).

77 Yeh/Liu, Committee Independence and Financial Institution Performance during the 2007-08 Credit Crunch: Evidence form a Multi-country Study, Corporate Governance: An International Review 2011, 19(5), 437.

78 Davies/Hopt, General Report, in Davies/Hopt/Nowak/van Solinge, eds., Corporate Boards in Law and Practice, A Comparative Analysis in Europe, Oxford 2013, p. 3 at 28 
dem Rechnung, Qualifikation und Sachkunde stehen klar im Vordergrund.79 Statt auf Unabhängigkeit in der Herkunft wird eher auf ,independent judgment" Wert gelegt. ${ }^{80}$

Die Anforderungen vor allem an Risk Management und Compliance ${ }^{81}$, aber auch an die Organisation und Arbeitsweise, namentlich durch Ausschüsse und insbesondere einen Risikoausschuss, ${ }^{82}$ sowie vor allem auch an die Vergütung und die Verhinderung von mit dieser verbundenen Fehlanreize $e^{83}$ und die besonderen Probleme in Bankkonzernen ${ }^{84}$ sind mittlerweile durch die nationalen und internationalen Gesetzgeber und konkretisierend durch Empfehlungen und Aufsichtspraxis für Organmitglieder von Banken und Finanzinstituten bis in kleinste Details neu geregelt und angehoben worden. Das alles ist deutlich

et seq., 34 et seq.; auch Davies/Hopt, Corporate Boards in Europe - Accountability and Convergence, The American Journal of Comparative Law 61 (2013) 301-375; auch Ferrarini/Filippelli, Independent Directors and Controlling Shareholders Around the World, ECGI Law Working Paper No. 258/2014, May 2014.

79 Z.B. Basel Committee (Fn. 1), Principle 2, auch wenn dann bei der Zusammensetzung die Rede ist von „a sufficient number of independent directors“, klar ist allerdings, dass die Direktoren keine Interessenkonflikte haben sollten, p. 3. Auch $\ 25 \mathrm{~d}$ KWG verlangt von den Mitgliedern des Verwaltungs- oder Aufsichtsorgans in erster Linie die erforderliche Sachkunde (unten Fn. 98). Aber z.B. FINMA (Fn. 8) p. 5: mindestens ein Drittel, Ausnahmen möglich. Auch Finesi ECFR 2015, 45.

80 FSB (Fn. 11) 4.5 p. 38 et seq.

81 Das Basel Committee (Fn. 1) widmet dem allein vier Principles von dreizehn. Die FINMA (Fn. 8) bündelt die Anforderungen an Corporate Governance, Risikomanagement und interne Kontrollen bei Banken. Zum Internal control framework mit einer Risk Management function ausführlich EBA (Fn. 9) p. 38 et seq., 45 et seq. Auch MÜlbeRT/WilHeLm ZHR 177 (2014) 502.

82 Z.B. $\iint 25$ d VII ff, VIII KWG, dazu Boos/Fischer/Schulte-Mattler (Fn. 35) $\$ 25 \mathrm{~d}$ KWG Rn $83 \mathrm{ff}, 96 \mathrm{ff}$.

83 Regeln und Literatur überborden, Nachweise allein zur deutschen Literatur bei MERKT/ KLAUSMANn EWS 2016, 253 (270 Fn. 212). International namentlich Financial Stability Forum (FSF), Principles for Sound Compensation Practices, 2009, und Principles for Sound Compensation Practives: Implementation Standards, 2009; Ferrarini (Fn. 19) p. 11 et seq. Empirische Befunde bei de HaAn/Vlahu (Fn. 19) 264 et seq., 266; HagenDORFF (Fn. 19) 143 et seq. Berichte bei Armour ET AL. (Fn. 4), p. 384 et seq., und Moore, Design and Control of Remuneration in UK Banks, in Chiu (Fn. 2) p. 133. Zu den Vorgaben der überarbeiteten Aktionärsrechterichtlinie (oben Rn. 47) für die Organvergütung (say on pay u. a.) BungerT/WANSLEBEN DB 2017, 1190, LEUERING NZG 2017, 646, Lanfermann/Maul BB 2017, 1218. Kritisch gegen einheitliche Regeln für die Vergütung im Bankensektor unter Hinweis auf die Disparitäten in verschiedenen Ländern VAN DER Elst (Fn. 19).

84 Z.B. Basel Committee (Fn. 1) Principle 5 p. 22 et seq.: Governance of group structures; FINMA (Fn. 8) No. 98, 99; Macey/O’Hara, Bank Corporate Governance: A Proposal for the Post-Crisis World, (2016) 22 Economic Policy Review (FRBNY) 85 at 90 et seq. (bank holding companies); MülbeRt/Wilhelm ZHR 178 (2014) 502 (530ff); SCHOCKENHOFF ZHR 180 (2016), 197. 
strenger als für Unternehmen allgemein, was zwar grundsätzlich berechtigt ist, aber insgesamt doch auf eine Überregulierung hinausläuft, die empirisch nicht fundiert ist. ${ }^{85}$ Besonderheiten ergeben sich schließlich - nach den Erfahrungen in der Finanzkrise sehr berechtigt ${ }^{86}$ aber mit klassischem Aktienrecht nicht zu vereinbaren und in der dortigen Diskussion zu Unrecht vernachlässigt - durch die Einbeziehung von Personal unterhalb der Organebene: sei es als eigene Position mit speziellen Aufgaben wie dem risk officer neben dem ComplianceBeauftragten oder einem eigenen Unternehmensbeauftragten für die Corporate Social Responsibility ${ }^{87}$, sei es allgemeiner Personen mit Schlüsselaufgaben (key function holders) mit eigenen Anforderungen bis hin zur Haftung. ${ }^{88}$

\section{Organisations- und Verbaltenspflichten, Haftung}

Die Organe von Banken und Finanzinstituten treffen durchweg strengere und insbesondere viel detaillierter und zwingend vorgegebene Organisations- und Verhaltenspflichten. ${ }^{89}$ Diese ergeben sich aus gesetzlichen Vorgaben, einer diese Vorgaben bis in die Details ausfächernden Aufsichtspraxis und weit ausholenden allgemeinen Corporate Governance-Prinzipien, die ihrer Rechtsnatur nach zwar rechtlich nicht bindend sind, aber tatsächlich doch weitgehend wie Rechtsnormen beachtet werden (und in der Praxis beachtet werden müssen). ${ }^{90}$ Der Einwand, solche Pflichten griffen wegen der üblichen rechtlichen Absicherung durch die Verwaltung (well-lawyered board) nicht, schlägt jedenfalls für Deutschland angesichts der hohen Anforderungen an Entlastung durch Rechtsrat nicht durch. ${ }^{91}$ Daraus resultieren durchaus andere Probleme

85 Kritik z.B. bei BINDER ZHR 2015, 667 (697f: fragwürdige Realbereichsanalyse, 707: kontroproduktiv); FERRARINI (Fn. 19) p. 2: für cautious deregelution, 24 et seq.

86 Nachweise bei Armour et AL. (Fn. 4), p. 383 et seq.

87 ReHBinder, FS Baums 2017, 959.

88 Basel Committee (Fn. 1) Principle 4: Senior management; EBA (Fn. 2) 54, klar weiter als nur der Vorstand; EBA (Fn. 9). Dazu Leyens/Schmidt AG 2013, 5334 (543ff). Umstrittene Ansätze für das klassische Aktienrecht durch die eigene Haftung des ComplianceBeauftragten, BGH NJW 2009, 3173.

89 Ausführlich BINDER ZGR 2015, 667; CHIU Journal of Business Law 2016/465; auch schon Merkt FS Hommelhoff 2012, 711. International Paolini, ed., Research Handbook on Directors' Duties, Cheltenham 2015. Gegen eine generelle Pflicht von Bankvorständen, bei ihren Entscheidungen auch die systemischen Gefahren mit zu berücksichtigen (public governance duty) zu Recht Hamdani (Fn. 19): too open-ended standard; Hamdani überlegt aber, systemische Risiken ähnlich wie self-dealing zu behandeln, also volle Transparenz und Zustimmung des ganzen board, eventuell auch nur der independent directors.

90 Zu letzteren vgl. oben Fn. 7-13.

91 BGH ZIP 2011, 2097 (Ision), kritisch m.w. N. Норт/Roth, GroßKommAktG 5. Aufl. $2015 \$ 93 \operatorname{Rdn} 139 f$. 
als bei der Corporate Governance von Nichtbanken, namentlich Rechtsunsicherheit wegen generalklauselartiger Prinzipien und ihrer Anwendung durch die Aufsicht in konkreten Fällen, Einschränkungen in der Geschäftstätigkeit, die ökonomisch auch aus Gläubigerschutzpostulaten nicht begründbar sind, zu wenig Flexiblität und Berücksichtigung von Besonderheiten (one size does not fit all) und Friktionen und Widersprüche zwischen Aufsichtsrecht und Gesellschaftsrecht. ${ }^{92}$

Die Umorientierung zu Creditor Goverance wirft auch zahlreiche Einzelfragen auf. Diese können dogmatischer Art sein wie vor allem die kontroverse Frage, ob aufsichtsrechtliche Verhaltenspflichten rein öffentlichrechtlich oder auch privatrechtlich zu verstehen sind, was erhebliche Konsequenzen für die Rechtsdurchsetzung hat. Während die Rechtsprechung und mehrheitlich die Literatur Ersteres annimmt, mehren sich die Vertreter der letzteren Ansicht, teilweise unter Berufung auf europarechtliche Rechtsdurchsetzungsvorgaben. ${ }^{93}$ Des weiteren fragt sich, ob auch bei Banken und Versicherungen ein Organisationsermessen des board bzw. der Leitungsorgane nach der Business Judgment Rule anerkannt wird..$^{94}$ Dieselbe Frage stellt sich aber auch funktional-rechtspolitisch und ökonomisch und wird unterschiedlich beantwortet, ist aber klar zu bejahen. ${ }^{95}$ Ganz unjuristisch ist die Vorstellung, für Bankdirektoren müsse ein anderer Sorgfaltspflichtmaßstab gelten, ${ }^{96}$ wenn nicht damit gemeint ist, dass der Gesetzgeber strengere Pflichten etwa für das Risikomanagement statuieren kann und statuiert. ${ }^{97}$ In die richtige Richtung geht dagegen die Aussage, dass von Bankdirektoren ein Mehr an Sachkunde

92 Auch Binder ZGR 2015, 667 (702ff).

93 Z.B. für die Verhaltenspflichten im Bank- und Kapitalmarktrecht Einsele ZHR 180 (2016) 233.

94 Bejahend unter $\$ 25$ a KWG (und ähnlich $\$ \$ 23,29$ VAG) z. B. Отт ZGR 2017, 149.

95 Für die business judgment rule auch unter der Corporate Governance von Finanzinstituten mit guten Gründen Ferrarini (Fn. 19) p. 21 et seq. unter Heranziehung von case law des Delaware Court of Chancery; auch Tröger (Fn. 19) und Hamdani (Fn. 19); dagegen Armour/Gordon, Systemic Harms and Shareholder Value, Journal of Legal Analysis 2014, 6 (35) p. 39 et seq. und schon ECGI Law Working Paper No. 222/2014, August 2014. Zur business judgment rule ausführlich Норт/Rотн in Großkommentar zum Aktienrecht, 5. Aufl., 2015, \$ 93 Rn 61-131.

96 Macey/O'Hara 2003 (Fn. 49) at 102 et seq.; dagegen näher Hopt (Fn. 6) 11.38 p. 353 et seq. Auch DERs., Responsibility of Banks and Their Directors, Including Liability and Enforcement, in Gorton/Kleineman/Wibom, eds., Functional or dysfunctional - the law as a cure? Risk and liability in the financial markets, Stockholm 2014, p. 159. Dass für die Bankdirektoren z.T. andere und schärfere Pflichten gelten, z.B. schon Litwin v. Allen, 25 NY2d 667 at 678 (Sup.Ct. 1940), ist etwas anderes als ein anderer Sorgfaltspflichtmaßstab, schon oben bei Fn. 96.

97 Dazu Mülbert/Wilhelm ZHR 178 (2014) 502 (507ff); für das UK Chiu, Corporate Governance and Risk Management in Banks and Financial Institutions, in Chiu (Fn. 2), p. 169. 
und Erfahrung verlangt werden muss ${ }^{98}$ - dies allerdings nicht undifferenziert für jedes einzelne Organmitglied, sondern zum Teil nur für das Organ in seiner Gesamtheit, ${ }^{99}$ sonst würde die notwendigen Diversität zu sehr eingeschränkt.

\section{4. Öffentlichrechtliche Finanzinstitute, insbesondere Landesbanken}

Einer gesonderten Behandlung bedürfen öffentlichrechtliche Finanzinstitute, in Deutschland insbesondere die Landesbanken. ${ }^{100}$ Das Basel Committee on Banking Supervision äußert sich dazu nur kursorisch. ${ }^{101}$ Die empirischen Befunde sind eindeutig. Maßgeblicher Einfluss der öffentlichen Hand geht einher mit negativen Auswirkungen auf die Qualität der Corporate Governance der Banken bzw. Finanzinstitute und auf ihre Ergebnisse. ${ }^{102}$ Das gilt besonders, wenn Organmitglieder weniger wegen ihrer Sachkunde als aus anderen Gründen bestellt werden. So hat eine empirische Studie zum Profil der 29 größten Banken während der Finanzkrise ergeben, dass öffentliche Banken, vor allem Landesbanken, zwischen dem ersten Vierteljahr 2007 bis zum dritten Vierteljahr 2008 dreimal höhere Verluste aufwiesen als Banken mit privaten Anteilseignern. Die zusätzliche Untersuchung der Biographien von

98 Macey/O’Hara 2016 (Fn. 84) at 86, 102 et seq., die eine „banking literacy“ ähnlich dem financial expert als Mitglied von audit committees unter dem Sarbanes-Oxlex Act fordern.

99 Richtig insoweit z.B. $\$ 25$ d Abs. 2 Satz 1 KWG: nur das Verwaltungs- oder Aufsichtsorgan in seiner Gesamtheit. Für das einzelne Organmitglied verlangt $\mathbb{2} 25 \mathrm{~d}$ Abs. 1 Satz 1 KWG „die erforderliche Sachkunde zur Wahrnehmung der Kontrollfunktion sowie zur Beurteilung und Überwachung der Geschäfte, die das jeweilige Unternehmen betreibt“. Zur persönlichen Qualifikation als Vorausssetzung auch bei Aufsichtsratsmitgliedern gemäß $\$ 24$ VAG (Schlüsselaufgabe) und zur Unterscheidung zwischen dem einzelnen Organmitglied und der Gesamtheit Hersch VersR 2016, 145 (147, 151, 146).

100 Vgl. die OECD Guidelines on Corporate Governance of State-Owned Enterprises, 19 November 2015 und dazu Hasche-Preusse WPg 2015, 1027; Bühler SZW 107 (2011) 513; aus rechtlicher Seite zur Corporate Governance in Aufsichtsräten von öffentlichen Unternehmen und zur Rolle von Public Corporate Governance Kodizes Weber-Rey/ Buckel ZHR 177 (2013) 13. Aus der Schweiz Chenaux/Rochat, Festschrift für von der Crone, 2017, 39.

101 Basel Committee (Fn. 1) Introduction No. 22 p. 7, nämlich nur: „The principles of sound corporate governance should also be applied to state-owned or state-supported banks, including when such support is temporary." (unter Verweisung auf die OECE Guidelines (Fn. 100).

102 de Haan/Vlahu (Fn. 19) 4.2 Government Ownerhsip, p. 263 et seq., 266, und z.B. Iannotta/Nocera/Sironi, Ownership structure, risk and performance in the European banking industry, Journal of Banking and Finance 31 (2007) 2127 at 2141, 2146 et seq. 
593 Aufsichtsratsmitgliedern dieser öffentlichen Banken ergab, dass ihre Erfahrung in Management und Finanzen systematisch niedriger lag als in den privaten Banken. Die Korrelation zwischen Verlusten und weniger Qualifikation und Erfahrung war statistisch hoch signifikant und legte Kausalität zwischen beidem nahe. ${ }^{103}$ Auch allgemeiner ist das Verhältnis von Staat und Unternehmen, namentlich Banken, problemträchtig und kann zu schädlichen Abhängigkeiten und Interaktionen führen. ${ }^{104}$ Für das Aufsichtsrecht muss das nicht notwendigerweise gelten, obwohl dort die seit langem bekannte Gefahr der regulatory capture besteht. ${ }^{105}$ Aber je mehr Einfluss dem Staat - auch nur in Gestalt einer heute zunehmend auch regulierenden Aufsicht - zugebilligt wird, und das ist bei der Corporate Goverance von Banken und Finanzinstituten der Fall, desto dringlicher wird die Frage nach den ökonomischen Auswirkungen, ganz unabhängig von politischen Einflussnahmen. ${ }^{106}$

\section{Durchsetzung und Kontrolle durch Staat und Private}

In der allgemeinen Corporate Governance-Diskussion wird der Frage der Durchsetzung und Kontrolle eine zentrale Bedeutung zugemessen. ${ }^{107}$ Das entspricht einer vermehrten Hinwendung in Literatur und Forschung von materiellrechtlichen Fragen im Aktienrecht zu verfahrens- und insolvenzrechtlichen Problemen. Im Bankrecht ist sogar von einer Wende vom Bankvertragsrecht hin zum Bankaufsichts- und -regulierungsrecht gesprochen worden. Nach den oben erörterten empirischen Befunden kann es hier gerade nicht um mehr Durchsetzung durch die Aktionäre ${ }^{108}$ gehen, namentlich Groß-

103 Hau/Thum, Subprime Crisis and Board (In-)Competence: Private vs. Public Banks in Germany, 21 June 2010, INSEAD Working Paper in Finance No. 2010/45/FIN, bei htt p://ssrn.com/abstract=1627921. Vgl. auch die harte Kritik von WOHLMANNSTETTER in Hopt/Wohlmannstetter (Fn. 4) p. 31, 47 et seq, 61 et seq.

104 Vgl. die Aufzählung bei Admati, Financialization and the Political Economy of Corporations, January 13, 2017, p. 10 et seq.

105 Dal Bó, Regulatory Capture: a Review, Oxford Review of Economic Policy 22(2) 2006, 203; Baxter, Capture in Financial Regulation: Can We Channel It Toward the Common Good? 21 Cornell Journal of Law \& Public Policy 2011, 175; Carpenter/ Moss, eds., Preventing Regulatory Capture: Special Interest Influence and How to Limit it, Cambridge 2013.

106 Der Sache nach auch LANGenbucher, ZHR 176 (2012) 652 (665): noch weitgehend unerforscht.

107 Einführend Hорт ZHR 175 (2011) 444 (465ff intern, $506 \mathrm{ff}, 514 \mathrm{ff}$ extern). Spezieller für Bankvorstände DERSELBE ZIP 2013, 1793, vor allem durch eine neue Verwaltung nach Ausscheiden der alten und durch Strafrecht. Zu securities law enforcement Anatomy (Fn. 2) 9.2 p. 258 et seq.

108 Ebenso Armour et Al. (Fn. 4), p. 388 et seq. Haftungsklagen durch Aktionäre sind aktienrechtlich selten, eher kapitalmarktrechtlich. International z.B. Klagen von Ak- 
aktionäre und institutionelle Anleger und Hedgefonds, die derzeit im Mittelpunkt der internationalen Corporate Goverance-Diskussion stehen, auch wenn die institutionellen Anleger durch bindende Verhaltenspflichten oder rechtlich nicht bindende Verhaltenskodices ${ }^{109}$ in Pflicht genommen werden. Aber der Schluss, an ihrer Stelle die Gläubiger in die Pflicht zu nehmen, greift zu kurz, denn die Probleme werden damit nur von einer Stakeholdergruppe zu einer anderen verschoben. Kleingläubiger wie Einleger haben ein rationales Desinteresse, zumal wenn sie durch die Einlagensicherung abgedeckt sind. ${ }^{110}$ Auch Schuldverschreibungsgläubiger haben nur begrenzt Potential und Interesse, die Corporate Governance von Emittenten zu beeinflussen und zu überwachen. ${ }^{111}$ Die international üblichen Covenants in den Anleihebedingung geben dafür kaum etwas her. ${ }^{112}$ Großgläubiger wie Banken halten verschiedenste Sicherheiten und haben deshalb außer im Vorfeld der Insolvenz in der Regel keinen Anreiz einzuschreiten. Die Deutschland-AG und das Hausbankensystem sind schon weithin abgebaut. ${ }^{113}$ Es bleibt die staatliche Aufsicht, die - funktional wie eine Treuhänderin für die debtholders und depositors für die Effektivität und Durchsetzbarkeit der Corporate Governance-Regeln sorgen und entsprechende Kompetenzen und Sanktionsmöglichkeiten haben muss ${ }^{114}$ und sich in technischer Hinsicht spezialisierter Bankwirtschaftsprüfer bedient. ${ }^{115}$ Dazu gehört auch die energische Anwendung der fit and proper-

tionären gegen die Royal Bank of Scotland wegen Irreführung der Aktionäre, beendet durch Vergleich.

109 Modellcharakter hat hier Financial Reporting Council, The UK Stewardship Code, September 2012. Zu berücksichtigen ist auch, dass die institutionellen Anleger am Kapitalmarkt nicht nur in Aktien, sondern auch in Obligationen investieren können.

110 Einlagensicherung wird deshalb in der ökonomischen Literatur vielfach für kontroproduktiv gehalten. Statt vieler Acharya/Anginer/Warburton, The end of market discipline? Investor expectation of implicit government guarantees, 1 May 2016, bei https://ssrn.com/abstract=1961656.

111 Норт (Fn. 4) at 243 zu den debtholders; Koккinis (Fn. 14) p. 25 et seq.

$112 \mathrm{Zu}$ den Anleihebedingungen ausführlich Oulds in Hopt/Seibt, Hrsg., Schuldverschreibungsrecht, 2017, S. $1174 \mathrm{ff}$, speziell zu den covenants Rn 3.44ff, zum change-ofcontrol Rn 3.82, zum cross default Rn 3.92 .

113 Ringe (Fn. 33).

114 FSB (Fn. 11) 2.3 p. 15 et seq.: Effectiveness and enforceability of corporate governance frameworks, mit Ausführungen zu den Kompetenzen und Sanktionen. Aus den USA z.B. enforcement rights der Federal Deposit Insurance Corporation (FIDIC), Atherton v. FIDIC, 519 U.S. 213 (1997). Zur (eingeschränkten) Rolle des Strafrechts Hopt (Fn. 107); Armour et AL. (Fn. 4), p. 390, mit Hinweis auf Financial Services (Banking Reform) Act (2013) s 36: criminal offence in case of reckless misconduct causing the firm to fail; Emmenegger, Hrsg., Bankrecht zwischen Strafrecht und Aufsichtsrecht, Basel 2014, zum Finanzmarktenforcement der FINMA darin eingehend WYss (von der FNMA), S. 83-139; für die USA auch MACEY/O'Hara 2016 (Fn. 84) at 101.

115 Zur eigenen Rolle der Aufsichtsbehörden speziell für die Corporate Governance praktisch alle eingangs erwähnten Institutionen (Fn. 7-13); zur Rolle von unabhängi- 
Anforderungen und der disqualification. ${ }^{116}$ Dass die Aufsicht den Board nicht gängeln, sondern ihm im Sinne einer Koregulierung Raum für eigenständige, interne Durchsetzung, geben sollte, ist wichtig. ${ }^{117}$ Eine Rolle können dabei auch eigene Verhaltenskodices für Banken - seien sie intern oder für die gesamte Branche - spielen, wie etwa der Dutch Banking Code zeigt ${ }^{118}$ und die von verschiedenen Institutionen wie dem Basel Committee, der EBA und der FSB besonders empfohlen werden. ${ }^{119}$ In Deutschland besteht hier ein klares Defizit. ${ }^{120}$ International sind die Erfahrungen mit der Durchsetzung von soft law durch die Nationalen Kontaktpunkte (NKP), wie sie die OECD mit ihren Vorschlägen zur Corporate Social Responsibility erfolgreich ins Spiel gebracht hat, interessant. Am Ende kommt es allerdings immer entscheidend auf Unternehmens- und Unternehmerethik und auf Unternehmenskultur an, die den tone from the top angeben muss, dies allgemein bei der Corporate Governance von Unternehmen, aber ganz besonders bei Banken und Finanzinstituten. ${ }^{121}$

\section{Zusammenfassung und Thesen}

Die Corporate Governance von Finanzinstituten weist gegenüber der allgemeinen Corporate Governance ganz erhebliche Besonderheiten auf, die durch empirische Befunde belegt werden und einen umfassenden Niederschlag in der mittlerweile überbordenden, nationalen und internationalen Finanzmarktgesetzgebung, in der Aufsichtspraxis und in vielen Prinzipien und Empfehlungen gefunden haben. Dem entspricht eine eigene Theorie der Corporate Governance von Finanzinstituten, die den Interessen der Gläubiger neben

gen, kompetenten und qualifizierten Prüfern z.B. FSB (Fn. 11) p. 5, 24 et seq. Zu den Abschlussprüfern als Finanzintermediären zwischen interner und externer Corporate Governance Leyens/Schmidt AG 2013, 533 (545f) und ausführlich Leyens (Fn. 42). Interessant Jавотіnsкy/Siems, How to Regulate the Regulators: Applying Principles of Good Corporate Governance to Financial Regulatory Institutions, ECGI Law Working Paper No 354/2017, May 2017.

116 Ernüchternder Bericht aus UK bei Lowry/Edmunds in Chiu (Fn. 2), p. 75.

117 Ferrarini (Fn. 19), schon im abstract. Vgl. Hodges, Law and Corporate Behaviour, Integrating Theories of Regulation, Enforcement, Complicance and Ethics, Oxford/ Portland 2015.

118 Oben Fn. 13.

119 Basel Committee on Banking Supervision (Fn. 1) p. 5: „Management should develop a written code of ethics or a code of conduct.“ EBA (Fn. 2) p. 31, 55; FSB (Fn. 11) p. 5 et seq. with recommendation 6, p. 30.

120 Hорт, A Plea for a Bankers' Code of Conduct, in Kenadjian/Dombret, eds., Getting the Culture and the Ethics Right, Towards a New Age of Responsiblity in Banking and Finnace, 2016, 75; kurz DERs., Audit Committee Quarterly III/2015, 20.

121 Zur Rolle des Unternehmenskultur z.B Financial Reporting Council, Corporate Culture and the Role of Boards: Report of Observations, July 2016. 
oder sogar vor denen der Aktionäre Rechnung trägt. Man kann insoweit von einer Creditor (Debtholder, Depositor) Governance sprechen. Diese hat erhebliche, noch nicht ausgelotete Folgen für Forschung und eventuelle Reformen, unter anderem für den Board bzw. Aufsichtsrat, für die Organisationsund Verhaltenspflichten seiner Mitglieder, für öffentlichrechtliche Finanzinstitute und für die Durchsetzung und Kontrolle durch Staat und Private.

Die Transparenz ist bei Finanzinstituten noch deutlich wichtiger als bei anderen Unternehmen, zumal sie zugleich der Aufsicht dient. Beim Board bzw. Aufsichtsrat ist die Unabhängigkeit der Mitglieder weniger wichtig als Sachkunde und Erfahrung mit Ausnahme der Prüfungs- und Risikoausschüsse. Eine ganz entscheidende Rolle kommt dem Risikomanagement und der Compliance zu und vor allem auch der Vergütung und der Verhinderung von mit dieser verbundenen Fehlanreizen. Dabei sind anders als im allgemeinen Aktienrecht auch Personen mit Schlüsselaufgaben (key function holders) einzubeziehen. Die Organe von Finanzinstituten treffen schon de lege lata strengere Organisations- und Verhaltenspflichten. Die business judgment rule muss aber auch für sie erhalten bleiben. Bei öffentlichrechtlichen Finanzinstituten, insbesondere Landesbanken sind keine Abstriche an Qualifikation und Erfahrung der Aufsichtsratsmitglieder zu dulden. Die Durchsetzung und Kontrolle muss für die Einleger die Aufsicht übernehmen. Dazu gehört die energische Anwendung der fit and proper-Anforderungen. 